\title{
Simultaneous Determination of 11 Preservatives in Cosmetics and Pharmaceuticals by Matrix Solid-phase Dispersion Coupled with Gas Chromatography
}

\author{
Yun-feng Liu*, Jia-ling Zhang, Xue-fei Nie, Ping Zhang, Xiao-qing Yan and Ke-feng Fu \\ School of Public Health, Shanxi Medical University, No. 56 Xinjian South Road, Taiyuan 030001, Shanxi, China
}

Received: 04 Sep 2019; accepted: 12 Sep 2019

\begin{abstract}
A convenient method was developed for simultaneous determination of 11 preservatives in cosmetics and pharmaceuticals. Matrix solid-phase dispersion had been optimized as the sample pretreatment technology, using Florisil as a dispersant, anhydrous sodium sulfate as a dehydrant, formic acid as an additive, and $n$-hexane and ethyl acetate as eluents successively, and followed by gas chromatography-flame ionization detection on a TR-5 capillary column. Experimental results showed that 11 preservatives were baseline separated within $22 \mathrm{~min}$. Good linearities were observed in the concentration range of $0.53-250 \mu \mathrm{g} / \mathrm{mL}$ for all analytes, and there were also minor differences. All correlation coefficients $(r)$ were more than 0.995 . The average recoveries at 3 levels of spiked samples ranged from $80 \%$ to $124 \%$ with $0.9-12 \%$ intra-day RSD and $1.8-12 \%$ inter-day RSD. The limits of detection were less than $0.18 \mu \mathrm{g} / \mathrm{mL}$ for all analytes. Besides, there was no obvious matrix effect on the analytes. The conclusion was that the developed method was simple, cheap, accurate, precise, and environment-friendly, in addition to existing little matrix effects. It could be recommended to determine 11 preservatives individually or in any their combinations to not only in liquid and gel cosmetics but also in liquid medicine and ointment.
\end{abstract}

Keywords: matrix solid phase dispersion, gas chromatography, cosmetics, pharmaceuticals, preservatives

\section{Introduction}

Synthetic preservatives, which can effectively suppress the growth of bacteria [1, 2], yeast [3], mold [3], and other microorganisms, are widely used as additives in daily necessities such as food, pharmaceuticals, cosmetics, and so on. The esters of $p$-hydroxybenzoic acid (parabens) have become the most frequently used preservatives, due to their strong stability, low volatility, and low irritability [4]. Iodopropynyl butylcarbamate used at low concentrations in cosmetics is an effective broad-spectrum fungicide [3]. Besides, phenoxyethanol and chlorphenesin are commonly employed in pharmaceuticals and cosmetics.

To prevent decay and extend the shelf life, proper amounts of preservatives can be added to some commodities, but excessive amounts will be toxic to human body. Parabens have some estrogenic activity, according to the toxicological study [5]. Some relevant studies reported that some parabens had been detected in human milk [6], urine [7], and human breast tumors [8]. Recently, people have paid attention to their safety and potential effects as the emerging pollutants, due to their potential endocrine disruption [2]. Consequently, the use of parabens in commodities has been regulated by national and trans-national departments or organizations. Meanwhile, researchers have been interested in developing sensible and reliable methods for their determination in products [2]. The antimicrobial activity of parabens is generally selective, so they are mixed with each other or other types of preservatives to increase antimicrobial activity and broaden the spectrum of anti-microorganisms [1].

A quick, convenient, economic, and effective method for determination of preservatives is needed to be developed, aim-

\footnotetext{
* Author for correspondence: lyfsl@163.com.
}

ing to ensure consumers' interests and provide references for relevant departments to set standards. Both cosmetics and pharmaceuticals are rarely simple matrices, containing multicomponents. For instance, cosmetic formulations are a mixture of surfactants, preservatives, sun screeners, fragrance, and so on. There are preservatives and ingredients in pharmaceuticals, in addition to components treating diseases. For most cosmetic and pharmaceuticals samples, if they were simply dissolved in solvent, the complex components would interfere with analytes and cause instrumental contamination. Thus, sample pretreatment procedures are vital. With regard to sample pretreatment for preservatives in some cosmetics and pharmaceuticals, most of them were simply dissolved with an appropriate organic solvent without further pretreatment except for vortex or filtration [9-15]. This method is very simple, but it will bring out interferences easily in analysis and require lots of toxic and harmful organic solvents, leading to lower efficiency and contaminations of equipment. To overcome these drawbacks, solid-phase extraction [16-19], ultrasonic-assisted extraction [20-23], and microextraction [24-25] have been recently studied.

Matrix solid-phase dispersion (MSPD), an extraction and purification technology, was first proposed by Barker et al. in 1989 [26]. The extraction and clean-up are completed simultaneously in a single step in this technique, consuming few solvents. It is a simple, quick, and effective method for using extraction and clean-up. More importantly, this method is particularly applied to solid, semi-solid, and highly viscous samples like cosmetics and pharmaceuticals. MSPD applied to cosmetics had been studied $[1,27]$, but they were followed by time-consuming derivatization and expensive instruments (gas chromatography-mass spectrometry [GC-MS] and ultra-performance liquid chromatography-mass spectrometry [UPLC-MS]), with the inevitable usage of hazardous solvents as eluents in UPLC.

This is an open-access article distributed under the terms of the Creative Commons Attribution-NonCommercial 4.0 International License (https://creativecommons.org/licenses/by-nc/4.0/), which permits unrestricted use, distribution, and reproduction in any medium for non-commercial purposes, provided the original author and source are credited, a link to the CC License is provided, and changes - if any - are indicated. 
In this work, a MSPD-GC method for extraction and determination of 11 preservatives in cosmetics and pharmaceuticals was developed. It is simple and quick and does not require derivatization. In the process of the sample treatment and detection, it is economic and environment-friendly and can help to reduce the solvent consumption.

\section{Experimental}

2.1. Chemicals and Reagents. Phenoxyethanol (PE), methylparaben (MP), ethylparaben (EP), propylparaben (PrP), and butylparaben (BuP) were purchased from Sigma-Aldrich (Shanghai) Trading Co., Ltd. (Shanghai, China). Isopropylparaben (iPrP) and phenylparaben $(\mathrm{PhP})$ were purchased from TCI (Shanghai) Development Co., Ltd. (Shanghai, China). Isobutylparaben (iBuP), benzylparaben (BzP), chlorphenesin, and iodopropynyl butylcarbamate (IPBC) were purchased from Aike Reagent Co., Ltd. (Chengdu, China), Shanghai Macklin Biochemical Co., Ltd. (Shanghai, China), Shanghai Aladdin Bio-Chem Technology Co., Ltd. (Shanghai, China), and Bailingwei Technology Co., Ltd. (Beijing, China), respectively. C18 $(50 \mu \mathrm{m})$ was obtained from Agela Technology Co., Ltd. (Tianjin, China). Florisil (60-100 mesh) was obtained from Sinopharm Chemical Reagent Co., Ltd. (Shanghai, China). Anhydrous sodium sulfate was obtained from Tianjin Kaitong Chemical Reagent Co., Ltd. (Tianjin, China). All the above chemicals were of analytical grade (purity $\geq 99 \%$ ). Methanol, acetonitrile, formic acid, ethyl acetate, and $n$-hexane were of HPLC-grade and provided by Tianjin Zhiyuan Chemical Reagent Co., Ltd. (Tianjin, China).

Florisil (selected 60-80 mesh by sieving) and anhydrous sodium sulfate were activated at $220{ }^{\circ} \mathrm{C}$ for $15 \mathrm{~h}$, and then, they were cooled down and kept in a desiccator.

2.2. Samples. Cosmetics and pharmaceuticals samples were purchased from the local supermarket and pharmacy.

2.3. Gas Chromatography-Flame Ionization Detection (GC-FID) Equipment. Gas chromatographic analyses were performed on a Thermo Scientific Trace 1300 system equipped with a split/splitless inlet, a FID detector, and an automatic injector. High-purity nitrogen $(>99.999 \%)$ supplied by high-pressure cylinders was used as carrier gas and makeup gas, and a HG-1803A high-purity hydrogen generator and an AG-1602 air generator (Beijing Keep-science Analysis Sci \& Tech Co. Ltd.) were used to supply hydrogen and air, respectively. The data was recorded and processed by Chromeleon 7.1 workstation software.

2.4. Sample Preparation. The procedure of MSPD was performed with some slight modifications on the basis of previously described method [26]. A 0.2 g dispersant (Florisil), a $0.15 \mathrm{~g}$ dehydration agent (anhydrous sodium sulfate), and a drop of formic acid (about $0.026 \mathrm{~g}$ ) were added to $0.05 \mathrm{~g}$ of cosmetic or pharmaceutical sample (exactly weight) in a Petri dish. Then, they were gently ground with a glass pestle for about 5 min to obtain a homogeneous mixture. A disposable plastic dropper was used as the solid-phase extraction column, of which part of the rubber bulb had been cut off and filled with a small amount of cotton and $0.05 \mathrm{~g}$ of Florisil successively at the pipette tip. Then, the obtained mixture was transferred into the column. Finally, the mixture was covered with $0.1 \mathrm{~g}$ of anhydrous sodium sulfate. The column was rinsed with $2 \mathrm{~mL}$ of $n$-hexane to remove grease and non-polar substances via gravity, collecting the fraction and measuring its volume as $V_{1}$ (approximately $1 \mathrm{~mL}$, another (2- $\left.V_{1}\right) \mathrm{mL}$ of $n$-hexane remained in the column). Then, elution with $3 \mathrm{~mL}$ ethyl acetate was performed to extract analytes, collecting the eluate (containing $\left(2-V_{1}\right) \mathrm{mL}$ of $n$-hexane). In order that the final solvent in the treated sample system was $n$-hexane-ethyl acetate $(1: 2, V / V$, the volume of $n$-hexane should be $1.67 \mathrm{~mL}$ for the total volume of $5 \mathrm{~mL}), 1.67-\left(2-V_{1}\right)$ $\mathrm{mL}$ of $n$-hexane was added, making up to $5 \mathrm{~mL}$ with ethyl acetate. The solution was directly analyzed by GC without any further treatment including filtration.

For the real samples, both cosmetics and medicine were processed as described above. In this study, cosmetics mainly included 5 different brands of liquid (\#1, \#2, and \#3 were toners, \#4 was a spray, and \#5 was lotion) and 3 different brands of gel cosmetics (\#1, \#2, and \#3 were Aloe vera gels). In addition, 2 different brands of liquid medicine (\#1 was spray and \#2 was eye drops) and 3 different brands of ointment (\#1 was emulsifiable paste, \#2 was oculentum, and \#3 was urea cream) were used.

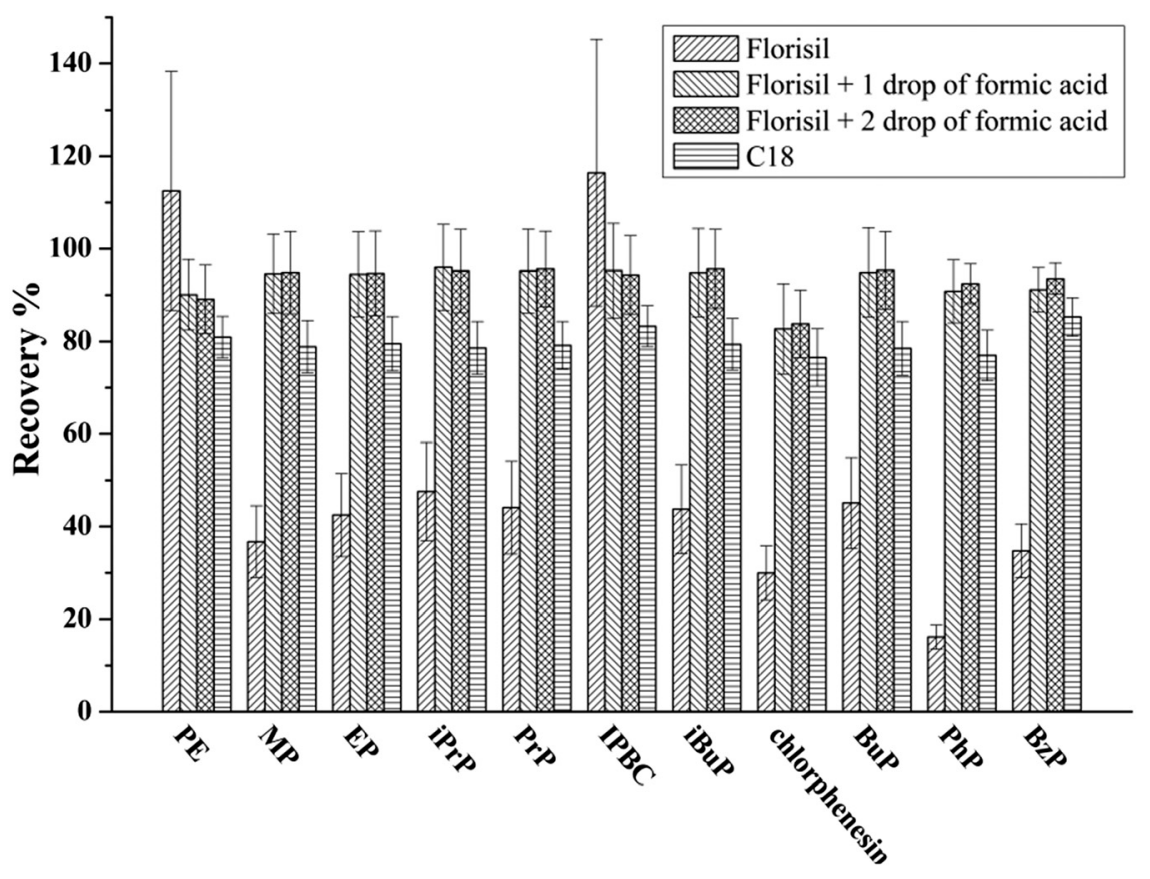

Figure 1. Type of dispersant (containing the test of adding formic acid) 


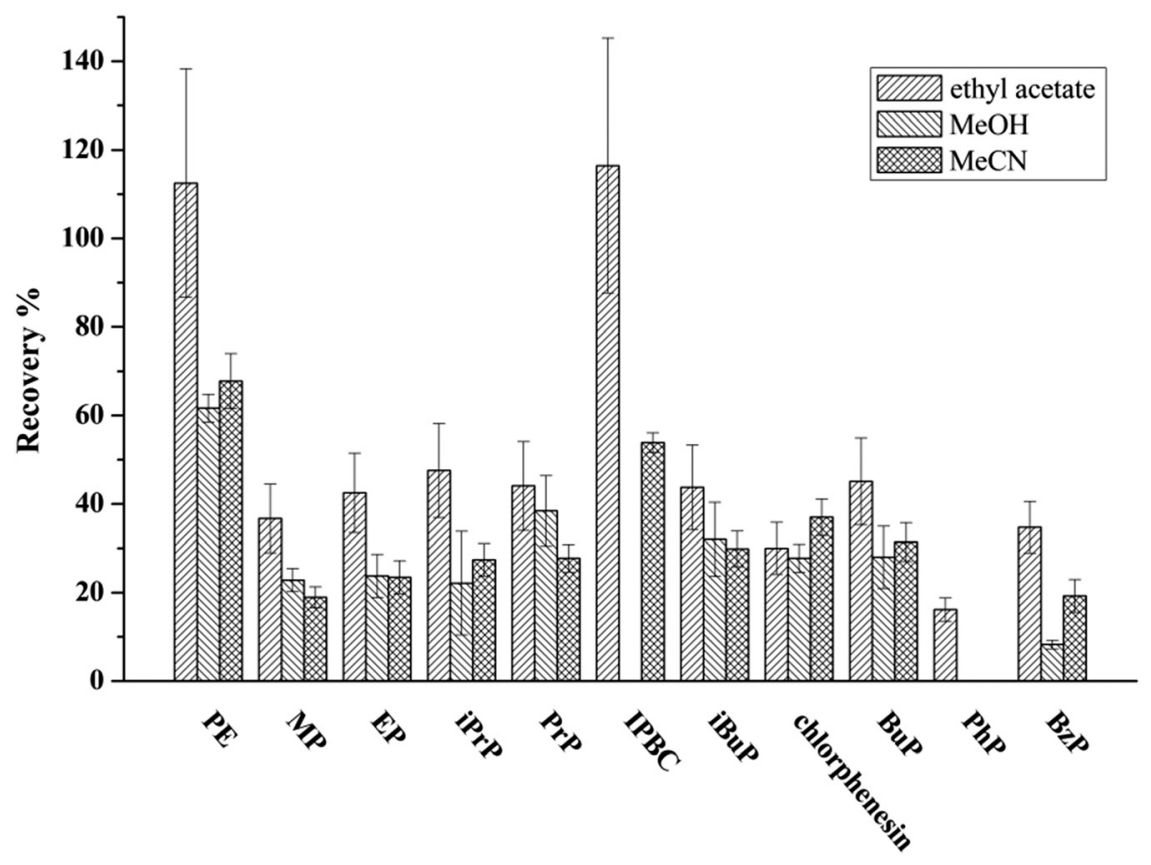

Figure 2. Type of elution solvent (not adding formic acid in this process)

2.5. GC Conditions. Column: the capillary column was TR-5 $(30 \mathrm{~m} \times 0.32 \mathrm{~mm} \times 0.25 \mu \mathrm{m})$; column temperature: $65^{\circ} \mathrm{C}(0 \mathrm{~min})$ to $100^{\circ} \mathrm{C}(1 \mathrm{~min})$ at $35^{\circ} \mathrm{C} / \mathrm{min}, 180^{\circ} \mathrm{C}(6 \mathrm{~min})$ at $10{ }^{\circ} \mathrm{C} / \mathrm{min}$, and $250^{\circ} \mathrm{C}(4 \mathrm{~min})$ at $50{ }^{\circ} \mathrm{C} / \mathrm{min}$; injection inlet temperature: $240{ }^{\circ} \mathrm{C}$; detector temperature: $280{ }^{\circ} \mathrm{C}$; carrier gas flow rate: $1.0 \mathrm{~mL} / \mathrm{min}$; hydrogen flow rate: $40 \mathrm{~mL} / \mathrm{min}$; air flow rate: $400 \mathrm{~mL} / \mathrm{min}$; makeup gas: $40 \mathrm{~mL} / \mathrm{min}$; split ratio: 1:10; and injection volume: $1 \mu \mathrm{L}$.

\section{Results and Discussion}

3.1. Optimization of MSPD Procedures. The efficiency of MSPD depends on various experimental factors. In this experiment, $10 \mu \mathrm{g} / \mathrm{mL}$ of mix standard solution was used to select the optimum condition including the type of dispersant, the type of elution solvent, and solvent ratios.
Two different dispersants (Florisil and C18) were tested, and the results listed in Figure 1. In this process, ethyl acetate was used as elution solvent. Compared with Florisil, higher recovery of most analytes was acquired using C18 as dispersant. However, with the removal of impurities, the analytes can be washed away by $n$-hexane. If a drop of formic acid was added, Florisil as the dispersant was chosen for the following studies, bringing to high clean-up degrees and satisfactory recoveries for all the analytes. Recoveries were still satisfactory, adding two drops of formic acid, but with worse inter-day precision. Thus, $0.2 \mathrm{~g}$ of Florisil as the dispersant material, a drop of formic acid as the additive, and $0.15 \mathrm{~g}$ of anhydrous sodium sulfate as the dehydrant were chosen to develop the extraction and clean-up method.

For the purpose of selecting the suitable elution solvent used to extract analytes in the process of sample preparation,

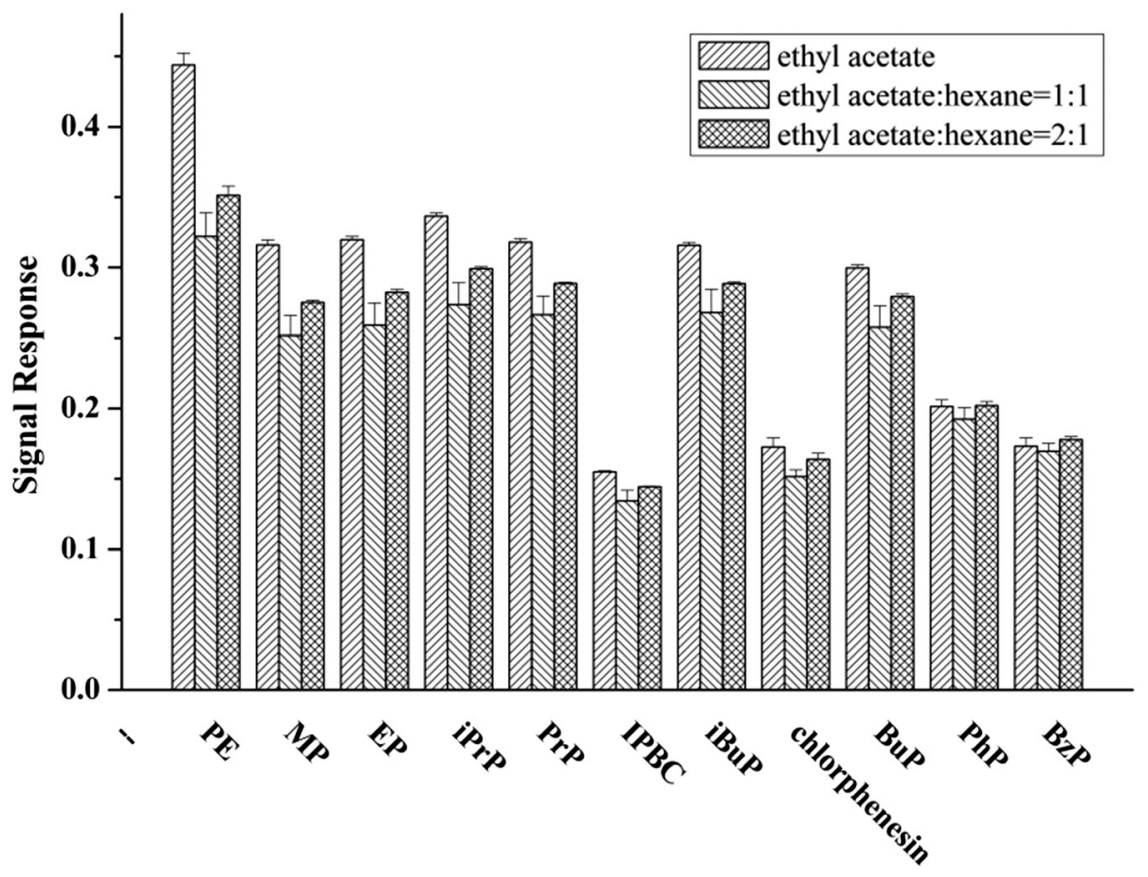

Figure 3. Solvent ratios (the volume ratio of ethyl acetate and hexane) 
Table 1. Regression equation, correlation coefficient (r), linear range, LOD, and LOQ of 11 preservatives in $n$-hexane-ethyl acetate (1:2, $V / V)$

\begin{tabular}{lcccc}
\hline Analytes & Regression equation & $r$ & Linear range $(\mu \mathrm{g} / \mathrm{mL})$ & $\mathrm{LOD}(\mu \mathrm{g} / \mathrm{mL})$ \\
\hline PE & $A=0.959 c+2.157$ & 0.9965 & $0.17 \sim 250$ & 0.056 \\
MP & $A=0.625 c+1.36$ & 0.9975 & $0.23 \sim 250$ & 0.076 \\
EP & $A=0.639 c+1.341$ & 0.9980 & $0.31 \sim 250$ & 0.10 \\
iPrP & $A=0.676 c+1.391$ & 0.9980 & $0.23 \sim 250$ & 0.17 \\
PrP & $A=0.666 c+1.238$ & 0.9985 & $0.16 \sim 250$ & 0.076 \\
IPBC & $A=0.332 c+0.862$ & 0.9980 & $0.53 \sim 250$ & 0.31 \\
iBuP & $A=0.680 c+1.117$ & 0.9990 & $0.20 \sim 250$ & 0.18 \\
Chlorphenesin & $A=0.470 c-0.242$ & 0.9995 & $0.41 \sim 250$ & 0.066 \\
BuP & $A=0.676 c+1.054$ & 0.9990 & $0.21 \sim 250$ & 0.14 \\
PhP & $A=0.762 c-0.822$ & 0.9985 & $0.32 \sim 250$ & 0.53 \\
BzP & $A=0.773 c-1.264$ & 0.9975 & $0.20 \sim 250$ & 0.20 \\
\hline
\end{tabular}

4 kinds of solvents including $n$-hexane ethyl acetate, methanol, and acetonitrile were tested by performing MSPD. The results were demonstrated in Figure 2. Ethyl acetate could provide the higher recovery for all the analytes except for chlorphenesin. In the test, the recovery did not rise and remained unchanged when the volume of ethyl acetate was more than $3 \mathrm{~mL}$. All the analytes were unable to be eluted by $n$ - hexane. Thus, $2 \mathrm{~mL}$ of $n$-hexane was used as the rinsing solvent to remove grease and non-polar substances other than the analytes, and then $3 \mathrm{~mL}$ of ethyl acetate was used as the elution solvent to elute and extract the analytes, namely, 11 preservatives.

Ethyl acetate, $n$-hexane-ethyl acetate $(1: 1, V / V)$ and $n$-hexane-ethyl acetate $(1: 2, V / V)$ were investigated. The results (Figure 3) showed that the signal response (peak area) was the

Table 2. Recoveries and precisions (intra-day RSD, inter-day RSD) (\%) of 11 preservatives in cosmetics and pharmaceuticals with MSPD-GC method $(n=6)$

\begin{tabular}{|c|c|c|c|}
\hline \multirow{2}{*}{$\begin{array}{l}\text { Sample types and } \\
\text { preservatives }\end{array}$} & \multicolumn{3}{|c|}{ The level of spiked standard solution } \\
\hline & Low & Medium & High \\
\hline \multicolumn{4}{|l|}{ Liquid cosmetic } \\
\hline $\mathrm{PE}$ & $103(3.6,2.4)$ & $110(5.8,9.7)$ & $102(2.5,4.6)$ \\
\hline MP & $94(2.0,3.0)$ & $102(5.8,6.5)$ & $87(5.3,2.9)$ \\
\hline EP & $85(1.8,2.8)$ & $97(6.7,5.8)$ & $97(7.2,2.7)$ \\
\hline iPrP & $80(2.1,2.9)$ & $98(6.8,6.1)$ & $101(5.1,2.7)$ \\
\hline PrP & $88(3.1,2.6)$ & $101(8.0,5.8)$ & $101(6.4,3.4)$ \\
\hline IPBC & $85(4.6,3.0)$ & $106(7.5,5.5)$ & $101(4.9,2.9)$ \\
\hline $\mathrm{iBuP}$ & $90(2.0,3.0)$ & $103(8.1,9.5)$ & $103(7.5,4.9)$ \\
\hline Chlorphenesin & $98(4.0,10.8)$ & $107(9.0,9.5)$ & $101(11.8,4.0)$ \\
\hline $\mathrm{BuP}$ & $87(2.4,3.8)$ & $101(8.8,5.8)$ & $102(8.1,4.5)$ \\
\hline $\mathrm{PhP}$ & $97(3.7,3.8)$ & $106(9.4,5.4)$ & $109(12,8.0)$ \\
\hline $\mathrm{BzP}$ & $104(3.3,3.8)$ & $113(7.8,5.3)$ & $107(11,6.5)$ \\
\hline \multicolumn{4}{|l|}{ Gel cosmetic } \\
\hline PE & $124(4.3,4.3)$ & $105(10,3.9)$ & $99(3.3,4.2)$ \\
\hline MP & $97(3.3,2.2)$ & $94(2.3,2.7)$ & $98(2.1,2.2)$ \\
\hline EP & $98(3.1,2.8)$ & $97(2.5,3.2)$ & $101(2.3,2.4)$ \\
\hline iPrP & $101(3.2,2.8)$ & $100(2.5,3.2)$ & $106(2.3,2.4)$ \\
\hline PrP & $103(3.2,3.7)$ & $101(2.9,3.8)$ & $107(2.8,2.8)$ \\
\hline IPBC & $110(4.7,2.7)$ & $109(2.3,4.3)$ & $117(2.2,2.1)$ \\
\hline $\mathrm{iBuP}$ & $106(3.0,4.2)$ & $104(3.1,4.2)$ & $110(3.1,3.0)$ \\
\hline Chlorphenesin & $122(3.4,8.8)$ & $107(13.1,7.1)$ & $115(6.8,4.5)$ \\
\hline $\mathrm{BuP}$ & $104(2.5,4.6)$ & $102(3.5,4.4)$ & $110(3.5,3.6)$ \\
\hline $\mathrm{PhP}$ & $105(1.7,7.5)$ & $104(5.2,5.1)$ & $108(10,7.3)$ \\
\hline $\mathrm{BzP}$ & $109(1.9,8.0)$ & $108(4.8,4.3)$ & $110(11,8.6)$ \\
\hline \multicolumn{4}{|l|}{ Liquid medicine } \\
\hline PE & $80(4.5,3.7)$ & $84(5.7,4.1)$ & $89(5.0,2.7)$ \\
\hline MP & $84(3.4,4.4)$ & $93(3.2,2.2)$ & $95(2.5,2.4)$ \\
\hline EP & $107(2.7,4.4)$ & $110(2.4,2.5)$ & $101(5.5,3.8)$ \\
\hline iPrP & $84(3.0,4.5)$ & $92(2.6,2.6)$ & $99(2.6,3.3)$ \\
\hline PrP & $88(3.2,7.0)$ & $100(1.8,4.4)$ & $104(6.3,3.8)$ \\
\hline IPBC & $91(4.5,4.9)$ & $100(1.9,3.4)$ & $110(2.9,4.2)$ \\
\hline iBuP & $91(3.2,7.4)$ & $102(0.9,4.9)$ & $106(3.1,4.7)$ \\
\hline Chlorphenesin & $88(4.8,8.3)$ & $86(3.4,4.9)$ & $94(4.2,4.8)$ \\
\hline $\mathrm{BuP}$ & $92(3.5,8.8)$ & $101(1.2,5.9)$ & $104(3.4,5.7)$ \\
\hline $\mathrm{PhP}$ & $114(3.2,9.8)$ & $113(2.9,8.3)$ & $110(4.7,7.4)$ \\
\hline \multirow{2}{*}{\multicolumn{4}{|c|}{ Ointment }} \\
\hline & & & \\
\hline PE & $83(2.8,4.1)$ & $91(4.0,3.6)$ & $92(4.0,3.7)$ \\
\hline MP & $93(2.4,1.8)$ & $102(3.2,3.1)$ & $104(2.9,3.2)$ \\
\hline EP & $88(3.1,2.1)$ & $97(3.6,3.9)$ & $99(2.8,3.3)$ \\
\hline iPrP & $91(3.4,1.9)$ & $103(3.8,3.9)$ & $99(2.9,3.4)$ \\
\hline PrP & $86(4.1,2.6)$ & $94(3.6,4.8)$ & $96(2.6,3.7)$ \\
\hline IPBC & $88(4.4,1.0)$ & $97(3.8,4.5)$ & $97(2.7,3.6)$ \\
\hline iBuP & $86(4.3,2.1)$ & $96(3.8,5.3)$ & $98(4.8,4.0)$ \\
\hline Chlorphenesin & $82(11.4,12)$ & $80(3.7,5.6)$ & $89(3.3,4.2)$ \\
\hline $\mathrm{BuP}$ & $87(5.5,2.3)$ & $96(3.7,5.7)$ & $99(3.3,4.1)$ \\
\hline $\mathrm{PhP}$ & $89(2.8,2.7)$ & $90(1.8,5.0)$ & $91(7.3,4.7)$ \\
\hline $\mathrm{BzP}$ & $92(4.8,0.8)$ & $92(2.5,4.8)$ & $93(8.3,4.8)$ \\
\hline
\end{tabular}

The concentration of spiked standard solution was 10,20 , and $40 \mu \mathrm{g} / \mathrm{mL}$ in liquid cosmetic; 26,40 , and $80 \mu \mathrm{g} / \mathrm{mL}$ in gel cosmetic; 5,10 , and $20 \mu \mathrm{g} / \mathrm{mL}$ in liquid medicine; and 5, 50 and $130 \mu \mathrm{g} / \mathrm{mL}$ in ointment. 


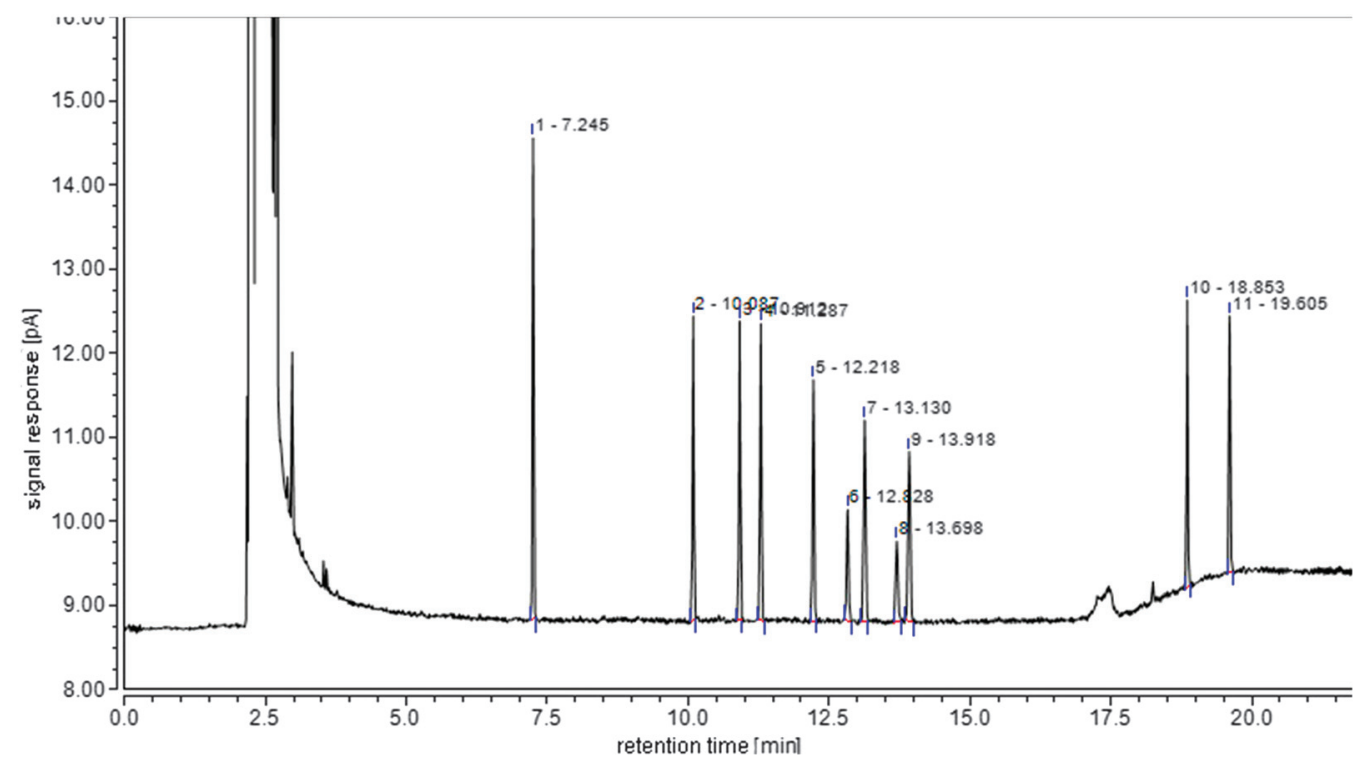

Figure 4. Chromatogram of analytes of $10 \mu \mathrm{g} / \mathrm{mL}$ of standard mixture solution in $n$-hexane-ethyl acetate $(1: 2, V / V)$ showing $1-11$ peak. Eleven preservatives and their corresponding retention time were as follows: (1) phenoxyethanol (PE), 7.245 min; (2) methylparaben (MP), 10.087 min; (3) ethylparaben (EP), $10.912 \mathrm{~min}$; (4) isopropylparaben (iPrP), $11.287 \mathrm{~min}$; (5) propylparaben (PrP), 12.218 min; (6) iodopropynyl butylcarbamate (IPBC), $13.828 \mathrm{~min}$; (7) isobutylparaben (iBuP), $13.130 \mathrm{~min}$; (8) chlorphenesin, $13.698 \mathrm{~min}$; (9) butylparaben (BuP), $13.918 \mathrm{~min}$; (10) phenylparaben (PhP), $18.853 \mathrm{~min}$; and (11) benzylparaben (BzP), $19.605 \mathrm{~min}$

highest using ethyl acetate as the solvent, and next to $n$-hexane-ethyl acetate $(1: 2, V / V)$ with better repeatability. In the experiment, the collected eluent was evaporated by a gentle stream of nitrogen at $25{ }^{\circ} \mathrm{C}$ and re-dissolved with $5 \mathrm{~mL}$ of ethyl acetate, resulting in the loss of analytes and the worse repeatability. Thus, $n$-hexane-ethyl acetate $(1: 2, V / V)$ was selected as the solvent.

In addition, the effects of the cotton and disposable plastic dropper were also investigated. The results showed that both cotton and disposable plastic dropper had little effect in the analysis. Moreover, they are extremely cheap and convenient.

Most of the materials used in this study are very simple, inexpensive, and harmless. Such a simple and cheap adsorbent as Florisil was selected as the dispersant. It can be used directly without further preparation and treatment except for simply sieving and activating at $220{ }^{\circ} \mathrm{C}$. Cotton is not as harmful as quartz wool, and it is also cheap. A disposable plastic dropper was used as the solid-phase extraction column, whose cost is far from the high-priced solid-phase extraction column. In addition, organic solvent consumption is low in the process of sample preparation (MSPD) and determination (GC).

3.2. Method Validation. To validate MSPD-GC method, a series of indexes including linear range, correlation coefficient, limit of detection (LOD), limit of quantification (LOQ), accuracy, and precision were studied.

A series of standard solution was analyzed, the calibration curve was given by Microsoft Excel 2007, and the corresponding regression equation can be expressed as $A=K c+b$, where $A$ is the GC signal response value (peak area) of each analyte and $c$ is the analyte concentration. LOD and LOQ were calculated using the formulas: $\mathrm{LOD}=3.3 \times \mathrm{SD} / K$ and $\mathrm{LOQ}=10 \times \mathrm{SD} / K$, respectively, where $\mathrm{SD}$ is the standard deviation of the peak height taken as a measure of noise, and $K$ is the slope of the corresponding calibration curve $(A=K c+b)$. The results are presented in Table 1.

Accuracy and precision were evaluated by recovery of standard addition and relative standard deviation (RSD) and determined by analyzing spiked samples at 3 concentration levels. Recoveries and intra-day precisions were calculated by analyzing mixed spiked sample in the same day $(n=6,6$ extractions performed in a day). Inter-day precision was determined by analyzing mixed spiked sample on 6 consecutive days (6 extractions performed in 6 days). The results were presented in Table 2.

The results demonstrated that the MSPD-GC method gave a good linearity and correlation coefficient, accuracy and precision.

The method was selective for simultaneous determination of 11 preservatives, and no interferences were observed at retention times corresponding to these analytes. Their chromatogram was demonstrated in Figure 4.

3.3. Matrix Effect [28, 29]. To know the matrix effect, 3 concentration levels (2, 40, and $160 \mu \mathrm{g} / \mathrm{mL}$ ) were investigated and each concentration level was tested in triplicate. The average responsible signal (peak area) of every component in solvent, which was $n$-hexane-ethyl acetate $(1: 2$, $V / V$ ), was marked as $A$. The corresponding peak areas for mixture standard solutions spiked after extraction into sample (cosmetic and pharmaceuticals) were marked as $B$. The matrix effect (ME) values can be calculated as follows: $\mathrm{ME}=B / A \times 100 \%$.

The values of more than $100 \%$ indicate signal enhancement, and the values of less than $100 \%$ indicate signal suppression. The results in Table 3 showed that matrix components in cosmetics and pharmaceuticals had no obvious effects on the signal responses of the analytes.

3.4. Application to Some Real Samples. The method presented was recommended to determine the target preservatives individually and in any their combinations in liquid and gel cosmetics and in pharmaceuticals, including liquid and ointment. All the detected samples were analyzed in triplicate, with quantitative analysis of the target preservatives according to an external standard curve. The contents of the preservatives determined in real samples were given in Table 4.

\section{Conclusions}

An effective, economic, and simple MSPD method coupled with GC was investigated to determine 11 preservatives in 
Table 3. Matrix effects of 11 preservatives in matrix dilutions of different sample extracts including liquid cosmetics, gel cosmetics, liquid medicine, and ointment

\begin{tabular}{|c|c|c|c|}
\hline \multirow{2}{*}{$\begin{array}{l}\text { Sample types and } \\
\text { preservatives }\end{array}$} & \multicolumn{3}{|c|}{ Concentrations $(\mu \mathrm{g} / \mathrm{mL})$} \\
\hline & 2 & 40 & 160 \\
\hline \multicolumn{4}{|l|}{ Liquid cosmetic } \\
\hline $\mathrm{PE}$ & $95.1 \pm 11$ & $95.6 \pm 1.2$ & $96.7 \pm 1.3$ \\
\hline MP & $111.2 \pm 6.5$ & $105.3 \pm 4.3$ & $105.5 \pm 4.7$ \\
\hline EP & $100.0 \pm 2.8$ & $107.1 \pm 4.8$ & $106.8 \pm 4.9$ \\
\hline iPrP & $103.5 \pm 4.2$ & $107.4 \pm 4.9$ & $107.1 \pm 5.1$ \\
\hline $\operatorname{PrP}$ & $108.2 \pm 6.2$ & $110.0 \pm 5.2$ & $108.8 \pm 5.0$ \\
\hline IPBC & $101.5 \pm 7.6$ & $107.3 \pm 4.5$ & $107.6 \pm 5.2$ \\
\hline $\mathrm{iBuP}$ & $113.5 \pm 4.1$ & $111.5 \pm 5.5$ & $109.3 \pm 5.0$ \\
\hline Chlorphenesin & $116.5 \pm 6.1$ & $120.7 \pm 5.8$ & $115.7 \pm 3.7$ \\
\hline $\mathrm{BuP}$ & $114.9 \pm 8.1$ & $112.3 \pm 5.6$ & $110.4 \pm 4.6$ \\
\hline $\mathrm{PhP}$ & $94.1 \pm 0.70$ & $114.3 \pm 3.0$ & $110.3 \pm 2.1$ \\
\hline $\mathrm{BzP}$ & $108.7 \pm 1.7$ & $114.7 \pm 3.8$ & $111.2 \pm 1.5$ \\
\hline \multicolumn{4}{|l|}{ Gel cosmetic } \\
\hline PE & $91.2 \pm 2.0$ & $98.0 \pm 3.0$ & $95.9 \pm 1.2$ \\
\hline MP & $119 . \pm 10.2$ & $104.8 \pm 10$ & $105.2 \pm 4.1$ \\
\hline EP & $103.7 \pm 3.7$ & $103.9 \pm 9.4$ & $105.5 \pm 4.0$ \\
\hline iPrP & $95.1 \pm 2.9$ & $104.2 \pm 9.7$ & $105.5 \pm 4.2$ \\
\hline $\operatorname{PrP}$ & $97.2 \pm 2.4$ & $106.0 \pm 10$ & $106.8 \pm 4.3$ \\
\hline IPBC & $99.1 \pm 4.5$ & $105.5 \pm 10$ & $105.9 \pm 4.2$ \\
\hline $\mathrm{iBuP}$ & $101.8 \pm 5.0$ & $106.5 \pm 10$ & $107.1 \pm 4.4$ \\
\hline Chlorphenesin & $84.4 \pm 10.6$ & $105.4 \pm 11.5$ & $106.7 \pm 3.9$ \\
\hline $\mathrm{BuP}$ & $111.4 \pm 5.8$ & $107.6 \pm 11$ & $107.7 \pm 4.1$ \\
\hline $\mathrm{PhP}$ & $96.8 \pm 7.3$ & $108.2 \pm 7.6$ & $105.9 \pm 2.9$ \\
\hline $\mathrm{BzP}$ & $103.2 \pm 8.7$ & $107.1 \pm 6.5$ & $104.8 \pm 2.3$ \\
\hline \multicolumn{4}{|l|}{ Liquid medicine } \\
\hline $\mathrm{PE}$ & $87.3 \pm 0.3$ & $99.3 \pm 2.9$ & $102.9 \pm 8.4$ \\
\hline MP & $97.0 \pm 3.7$ & $102.8 \pm 1.1$ & $107.5 \pm 4.8$ \\
\hline EP & $121.0 \pm 8.5$ & $104.9 \pm 1.9$ & $108.4 \pm 4.5$ \\
\hline iPrP & $97.6 \pm 3.8$ & $103.2 \pm 1.4$ & $107.9 \pm 4.4$ \\
\hline $\operatorname{PrP}$ & $99.8 \pm 2.6$ & $104.6 \pm 2.3$ & $108.0 \pm 3.6$ \\
\hline IPBC & $100.9 \pm 2.7$ & $103.8 \pm 2.0$ & $107.8 \pm 4.2$ \\
\hline $\mathrm{iBuP}$ & $103.3 \pm 2.4$ & $105.1 \pm 2.6$ & $107.8 \pm 3.3$ \\
\hline Chlorphenesin & $95.4 \pm 15.1$ & $98.2 \pm 4.3$ & $103.8 \pm 1.9$ \\
\hline $\mathrm{BuP}$ & $106.5 \pm 3.6$ & $105.5 \pm 3.1$ & $107.9 \pm 2.8$ \\
\hline $\mathrm{PhP}$ & $92.8 \pm 6.7$ & $107.3 \pm 2.9$ & $102.7 \pm 2.1$ \\
\hline $\mathrm{BzP}$ & $102.0 \pm 2.4$ & $106.6 \pm 2.8$ & $101.5 \pm 2.4$ \\
\hline \multicolumn{4}{|l|}{ Ointment } \\
\hline $\mathrm{PE}$ & $93.1 \pm 3.5$ & $100.0 \pm 2.7$ & $96.4 \pm 1.7$ \\
\hline MP & $102.6 \pm 4.7$ & $100.8 \pm 2.3$ & $98.7 \pm 3.1$ \\
\hline $\mathrm{EP}$ & $98.3 \pm 5.3$ & $101.2 \pm 2.9$ & $99.2 \pm 3.6$ \\
\hline iPrP & $99.4 \pm 2.0$ & $101.2 \pm 3.0$ & $99.2 \pm 3.7$ \\
\hline $\operatorname{PrP}$ & $103.1 \pm 5.3$ & $102.5 \pm 3.7$ & $100.4 \pm 4.4$ \\
\hline IPBC & $100.9 \pm 2.7$ & $101.8 \pm 3.2$ & $99.4 \pm 3.9$ \\
\hline $\mathrm{iBuP}$ & $103.9 \pm 5.6$ & $103.3 \pm 4.0$ & $100.6 \pm 4.5$ \\
\hline Chlorphenesin & $117.7 \pm 7.2$ & $106.4 \pm 5.2$ & $103.9 \pm 5.5$ \\
\hline $\mathrm{BuP}$ & $106.7 \pm 2.8$ & $103.8 \pm 4.1$ & $101.6 \pm 4.9$ \\
\hline $\mathrm{PhP}$ & $110.7 \pm 6.8$ & $107.7 \pm 4.5$ & $104.2 \pm 5.1$ \\
\hline $\mathrm{BzP}$ & $105.2 \pm 6.7$ & $106.8 \pm 4.1$ & $103.6 \pm 4.7$ \\
\hline
\end{tabular}

cosmetics and pharmaceuticals. MSPD uses a single treatment step and this method is simple and rapid in extraction and purification. The optimized MSPD method does not require expensive equipment or instruments and a large amount of organic solvent. Furthermore, compared with derivatization or MS, it is time-saving, environment-friendly, and cheap.

On the whole, the proposed method could be applied to determine the preservatives in liquid and gel cosmetics and

Table 4. Analytical results of the analytes in real samples using the proposed MSPD-GC technique

\begin{tabular}{|c|c|c|c|c|c|c|c|c|c|c|c|c|}
\hline \multirow[t]{2}{*}{ Sample } & & \multicolumn{11}{|c|}{ Content, $\%$} \\
\hline & & PE & MP & EP & iPrP & PrP & IPBC & $\mathrm{iBuP}$ & Chlorphenesin & $\mathrm{BuP}$ & $\mathrm{PhP}$ & $\mathrm{BzP}$ \\
\hline \multirow{6}{*}{ Liquid cosmetics } & $\# 1$ & 0.182 & 0.063 & - & - & - & - & - & - & - & - & - \\
\hline & $\# 2$ & 0.314 & 0.138 & - & - & 0.042 & - & - & - & - & - & - \\
\hline & \#3 & 0.372 & 0.051 & - & - & - & - & - & - & - & - & - \\
\hline & $\# 4$ & - & - & - & - & - & - & - & - & - & - & - \\
\hline & $\# 5$ & 0.257 & 0.067 & - & - & - & - & - & - & - & - & - \\
\hline & $\# 1$ & 0.375 & - & - & - & - & - & - & 0.224 & - & - & - \\
\hline \multirow[t]{2}{*}{ Gel cosmetics } & $\# 2$ & 0.99 & - & - & - & - & - & - & - & - & - & - \\
\hline & $\# 3$ & - & - & 0.059 & - & - & - & - & - & - & - & - \\
\hline \multirow{3}{*}{ Liquid medicine } & $\# 1$ & - & - & - & - & - & - & - & - & - & - & - \\
\hline & $\# 2$ & - & - & - & - & 0.046 & - & - & - & - & - & - \\
\hline & $\# 1$ & - & - & - & - & - & - & - & - & - & - & - \\
\hline \multirow[t]{2}{*}{ Ointment } & $\# 2$ & - & - & - & - & - & - & - & - & - & - & - \\
\hline & $\# 3$ & - & 0.081 & - & - & - & - & - & - & - & - & - \\
\hline - Not detected. & & & & & & & & & & & & \\
\hline
\end{tabular}


liquid medicine and ointment. Not only can it provide good recoveries and precisions, but also it has little matrix effect for determination of target preservatives.

\section{References}

1. Sanchez-Prado, L.; Alvarez-Rivera, G.; Lamas, J. P.; Lores, M.; GarciaJares, C.; Llompart, M. Anal. Bioanal. Chem. 2011, 401, 3293.

2. Ocaña-González, J. A.; Villar-Navarro, M.; Ramos-Payan, M.; Fernandez-Torres, R.; Bello-López, M. A. Anal. Chim. Acta. 2015, 858, 1.

3. Palmer, K. B.; LaFon, W.; Burford, M. D. J. Chromatogr. A. 2017, 1516, 131.

4. Aoyama, A.; Doi, T.; Tagami, T.; Kajimura, K. J. Chromatogr. Sci. 2014, 52,1010 .

5. Routledge, E. J.; Parker, J.; Odum, J.; Ashby, J.; Sumpter, J. P. Toxicol. Appl. Pharmacol. 1998, 153, 12.

6. Schlumpf, M.; Kypke, K.; Wittassek, M.; Angerer, J.; Mascher, H.; Mascher, D.; Vökt, C.; Birchler, M.; Lichtensteiger, W. Chemosphere. 2010 81,1171 .

7. Meeker, J. D.; Yang, T.; Ye, X. Y; Calafat, A. M.; Hauser, R. Environ. Health. Persp. 2011, 119, 252.

8. Darbre, P. D.; Aljarrah, A.; Miller, W. R.; Coldham, N. G.; Sauer, M. J.; Pope, G. S. J. Appl. Toxicol. 2004, 24, 5.

9. Noguera-Ortí, J. F.; Villanueva-Camañas, R. M.; Ramis-Ramos, G. J. Chromatogr. Sci. 1999, 37, 83.

10. He, S.; Zhao, Y.; Zhu, Z.; Liu, H.; Li, M.; Shao, Y.; Zhuang, Q. Talanta. 2006, 69, 166 635.

11. Memon, N.; Iqbal-Bhanger, M.; Khuhawer, M. Y. J. Sep. Sci. 2005, 28 ,

12. Huang, J. Q.; Hu, C. C.; Chiu, T. C. Int. J. Cosmetic. Sci. 2013, 35,
13. Haunschmidt, M.; Buchberger, W.; Klampfl, C. W.; Hertsens, R. Anal. Methods. 2011, 3, 99.

14. Ballesta-Claver, J.; Valencia, M. C.; Capitán-Vallvey, L. F. Talanta. 2009, 79, 499

15. Wang, Y.; Cao, Y. H.; Fang, C.; Gong, Q. Q. Anal. Chim. Acta. 2010, 673,145

16. González-Mariño, I.; Quintana, J. B.; Rodríguez, I.; Cela, R. Rapid. Commun. Mass. Sp. 2009, 23, 1756.

17. Uysal, U. D.; Güray, T. J. Anal. Chem. 2008, 63, 982.

18. Ballesta-Claver, J.; Valencia, M. C.; Capitán-Vallvey, L. F Luminescence. 2011, 26, 44.

19. Márquez-Sillero, I.; Aguilera-Herrador, E.; Cárdenas, S.; Valcárcel, M. J. Chromatogr. A. 2010, 1217,1 367.

20. Wu, T.; Wang, C.; Wang, X.; Ma, Q. Int. J. Cosmetic. Sci. 2008, 30 ,

21. Huang, H. Y.; Lai, Y. C.; Chiu, C. W.; Yeh, J. M. J. Chromatogr. A. 2003, 993, 153.

22. Núñez, L.; Tadeo, J. L.; García-Valcárcel, A. I.; Turiel, E. J. Chromatogr. A. 2008, 1214, 178.

23. Yamini, Y.; Saleh, A.; Rezaee, M.; Ranjbar, L.; Moradi, M. J. Liq. Chromatogr. R. T. 2012, 35, 2623.

24. Farajzadeh, M. A.; Khosrowshahi, E. M.; Khorram, P. J. Sep. Sci. 2013, 36, 3571

25. Fei, T.; Li, H. F.; Ding, M. Y.; Ito, M.; Lin, J. M. J. Sep. Sci. 2011, 34, 1599.

26. Barker, S. A.; Long, A. R.; Short, C. R. J. Chromatogr. A. 1989, 475, 353.

27. Djatmika, R.; Ding, W. H.; Sulistyarti, H. IOP Conf Ser: Mater. Sci. Eng. 2018, 299, 012005.

28. Matuszewski, B. K.; Constanzer, M. L.; Chavez-Eng, C. M. Anal. Chem. 2003, 75, 3019.

29. Liu, S.; Ying, G. G.; Zhao, J. L.; Chen, F.; Yang, B.; Zhou, L. J.; Lai, H. J. J. Chromatogr. A. 2011, 1218, 1367. 